\title{
The mathematical description for the electropolymerization of furan, pyrrole and thiophene derivatives in alkaline media
}

\author{
Volodymyr Tkach*, Vasyl’ Nechyporuk and Petro Yagodynets' \\ Chernivtsi National University, Ukraine
}

\begin{abstract}
The electropolymerization of furan, pyrrole and thiophene in strong basic media was mathematically described. Two models for the cases of the insoluble and soluble anodes were described and analyzed by using the linear stability theory and bifurcation analysis and compared with the models for other cases of electropolymerization. The causes for the oscillatory behavior, monotonic instabilities and steady-state stability conditions were found.
\end{abstract}

Keywords: conducting polymers; corrosion protection; alkaline media; electrochemical oscillations; stable steady-state.

\section{Introduction}

Conducting polymers, being one of the most studied materials during the 5 last decades $^{1-20}$, attract more and more attention, due to their capability to combine the properties of plastics (corrosion stability, tough, light weight, resilience and versatility in shaping), metal conductivity and facility of modification. This gives them the possibility for the vast and rich spectrum of use: from the corrosion-protecting coatings to sensors and biosensors.

Conducting polymers may be obtained chemically and electrochemically, but electrochemically obtained CPs have some advantages, compared to the chemosynthetized (they are more conductive, because of the coplanarity of monomer rings and being obtained in doped form $)^{1-5}$.

The properties of conducting polymers (either physical - ability for corrosion protection, morphology, film thickness, or chemical) depend strongly on the conditions, in which they are synthetized. For example, strongly acid media, in general, favors the electropolymerization, but damages the morphology of the resulting polymer (by proton attacks, acidofobic side reactions etc.). Strongly basic media affects the electropolymerization by entering of hydroxyl as a dopant in the polymer matrix ${ }^{6-10}$. Also, if the anode material is soluble in alkali, it causes its corrosion and the conducting polymer coating serves as protector.

In general, monomer polymerization potential may or may not be higher than the potential of hydroxide oxidation:

$$
4 \mathrm{OH}^{-}-4 \mathrm{e}^{-} \longrightarrow \mathrm{O}_{2}+2 \mathrm{H}_{2} \mathrm{O}
$$


So, the influence of this process has also to be studied, depending on polymerization potential and mobility of hydroxyl ions in the solvent.

Overmore, either for anodic electropolymerization processes ${ }^{10-20}$, or for metal anodic dissolution processes (the sample system is $\left.\mathrm{Fe}-\mathrm{H}_{2} \mathrm{SO}_{4}\right)^{21-23}$ the electrochemical instabilities, like oscillatory behavior and monotonic instability, are characteristic.

The authors of the works ${ }^{10,14,16,18}$ observed the oscillations in potential during the electropolymerization of thiophene and pyrrole in different media. In the case of thiophene ${ }^{10}$, the oscillation amplitude was dependent of the dopant used (which may give the supposal of the factors of double electric layer (DEL), influencing this process) and the polymer morphology was dependent of the distance between the current thermodynamic state of the polymer of the equilibrium, which gave to the authors the reason to suppose the presence of the surface instability in the electropolymerization process. In the other works ${ }^{11,12,15,17}$, the oscillatory behavior in potential was associated with corrosion processes. Oscillations in current have also been observed in ${ }^{13}$ for indole and in ${ }^{20}$ for thiophene.

They have gained their phenomenological explanation in terms of the experimental investigation, but such an explanation has a serious lack of fundamentality, which may be resolved by developing a mathematical model, capable to describe adequately the processes in the system. It is also capable to offer the explanation, valid not only for each concrete system, but also for the similar ones.

Various efforts have been made for our group in describing different electropolymerization systems in neutral and strongly acid media ${ }^{25-34}$. In this work, we describe three models for the different cases of electropolymerization of one heterocyclic compound in strongly basic (alkaline) media.

\section{System and its modeling}

For us to describe the electropolymerization of a heterocyclic monomer in alkaline media, we introduce 3 variables:

$\mathrm{c}$ - the monomer concentration in pre-surface layer;

$\theta$ - the monomer coverage degree;

$\mathrm{a}$ - the alkali concentration in pre-surface layer.

In order to simplify the modeling, we suppose that the reactor is intensively stirred, so we can neglect the convection flow. Also we suppose that the background electrolyte (containing the main dopant) is in excess, so we can neglect the migration flow. The concentration profile of the monomer and the alkali in pre-surface layer (with constant thickness, equal to $\delta$ ) is supposed to be linear.

The monomer enters the pre-surface layer by diffusion and desorption from the electrode surface and leaves it by specific adsorption. So, the balance equation for the monomer concentration may be written like:

$$
\frac{d c}{d t}=\frac{2}{\delta}\left(\frac{\Delta}{\delta}\left(c_{0}-c\right)+r_{-1}-r_{1}\right)
$$

in which $\Delta$ is the monomer diffusion coefficient, $\mathrm{r}_{1}$ and $\mathrm{r}_{-1}$ are adsorption and desorption rates, $\mathrm{c}_{0}$ is the monomer bulk concentration.

The monomer enters the surface by adsorption, leaving it by desorption. Its surface concentration also diminishes by electropolymerization. So, the balance equation will be rewritten as: 


$$
\frac{d \theta}{d t}=\frac{1}{\Gamma_{\max }}\left(r_{1}-r_{-1}-r_{2}\right)
$$

in which $\Gamma_{\max }$ is monomer maximal surface concentration and $\mathrm{r}_{2}$ the electropolymerization rate.

The alkali enters the pre-surface layer by diffusion and leaves it by electropolymerization, in which it takes part as a dopant. So, its balance equation will be rewritten as:

$$
\frac{d a}{d t}=\frac{2}{\delta}\left(\frac{D}{\delta}\left(a_{0}-a\right)-r_{2}\right)
$$

in which $\mathrm{a}_{0}$ stands for alkali bulk concentration and $\mathrm{D}$ for its diffusion coefficient. In the case of the participation of hydroxyl in other reactions (like metal or film dissolution, anodic oxidation), the equation (4) suffers changes, described lower.

The rates of the processes, described above are calculated as following:

$$
\begin{aligned}
& r_{1}=k_{1} \exp \left(-\frac{\left(K_{0}-K_{1}\right) \varphi_{0}^{2}+2 \mathrm{~K}_{2} \varphi_{0} \varphi_{1}}{2 R T \Gamma_{\max 1}} \gamma\right) \exp (a \theta) c(1-\theta), \\
& r_{-1}=k_{-1} \exp \left(\frac{\left(K_{0}-K_{1}\right) \varphi_{0}^{2}+2 \mathrm{~K}_{2} \varphi_{0} \varphi_{1}}{2 R T \Gamma_{\max 1}}(1-\gamma)\right) \exp (-a \theta) \theta, \\
& r_{2}=k_{2} a^{j} \theta^{x} \exp \left(-\frac{z F}{R T} \varphi_{0}\right)
\end{aligned}
$$

In which $\mathrm{k}_{\mathrm{x}}$ are rate constants of respective processes, $\varphi_{0}$ and $\varphi_{1}$ are potential slopes in DEL, corresponding to the free and monomer-occupied surface parts, $\gamma$ is the constant, $\mathrm{K}_{0}$ and $\mathrm{K}_{1}$ are integral capacitances of the parts of DEL, corresponding to the free and monomeroccupied surface parts, $\mathrm{F}$ is Faraday number, $\mathrm{z}$ is the number of electrons transferred during the electropolymerization, $\mathrm{j}$ and $\mathrm{x}$ are reaction orders of alkali and the monomer in electropolymerization reactions.

\section{Results and Discussion}

The behavior of this system is analyzed by linear stability theory and bifurcation analysis. The steady-state Jacobian members may be represented as:

$$
\left(\begin{array}{lll}
a_{11} & a_{12} & a_{13} \\
a_{21} & a_{22} & a_{23} \\
a_{31} & a_{32} & a_{33}
\end{array}\right)
$$


in which:

$$
\begin{array}{ccc}
a_{11}=\frac{\partial F_{1}}{\partial c}=\frac{2}{\delta}\left(-\frac{\partial r_{1}}{\partial c}-\frac{\Delta}{\delta}\right) & a_{12}=\frac{\partial F_{1}}{\partial \theta}=\frac{2}{\delta}\left(-\frac{\partial r_{1}}{\partial \theta}+\frac{\partial r_{-1}}{\partial \theta}\right) & a_{13}=\frac{\partial F_{1}}{\partial a}=0 \\
a_{21}=\frac{\partial F_{2}}{\partial c}=\Gamma_{\max 1}^{-1} \frac{\partial r_{1}}{\partial c} & a_{22}=\frac{\partial F_{2}}{\partial \theta}=\Gamma_{\max 1}^{-1}\left(\frac{\partial r_{1}}{\partial \theta}-\frac{\partial r_{-1}}{\partial \theta}-\frac{\partial r_{2,}}{\partial \theta} a_{23}=\frac{\partial F_{2}}{\partial a}=\Gamma_{\max 1}^{-1}\left(-\frac{\partial r_{2,}}{\partial a}\right)\right. \\
a_{31}=\frac{\partial F_{3}}{\partial c}=0 & a_{32}=\frac{\partial F_{3}}{\partial \theta}=-\frac{\partial r_{2,}}{\partial \theta} & a_{33}=\frac{\partial F_{3}}{\partial a}=\frac{2}{\delta}\left(-\frac{\partial r_{2}}{\partial a}-\frac{D}{\delta}\right)
\end{array}
$$

To make Jacobian analysis more easy and avoid the appearance of complicated expressions, we introduce new variables in the purpose of Jacobian determinant to obtain the next form:

$$
\frac{4}{\delta^{2} \Gamma_{\max }}\left|\begin{array}{ccc}
-\kappa_{1}-\xi & -X_{1} & 0 \\
\xi & X_{1}-X_{2} & -\omega \\
0 & -X_{2} & -\omega-\kappa_{2}
\end{array}\right|
$$

in which $\kappa_{1}=\frac{\Delta}{\delta}$ and $\kappa_{2}=\frac{D}{\delta}$ are diffusion Jacobian parameters, $\xi=\frac{r_{1}}{c}$ and $X_{1}=$ $\frac{\partial r_{1}}{\partial \theta}-\frac{\partial r_{-1}}{\partial \theta}$ are surface behavior parameters (describing adsorption and desorption), $X_{2}=\frac{\partial r_{2}}{\partial \theta}$ is electropolymerization parameter and $\omega=\frac{\partial r_{2,}}{\partial a}$ is the parameter of participation of an alkali in the polymerization process.

Using the Routh-Hurwitz steady-state stability criterion's main requirement (Det $\mathrm{J}<0$ ), we obtain the steady-state stabilidy condition for the simplest case of the electropolymerization of a heterocyclic compound in alkaline media in the form of an inequality, resolved by alkali diffusion parameter $\kappa_{2}$ :

$$
\kappa_{2}<\frac{X_{2} \kappa_{1}+X_{2} \xi-X_{1} \kappa_{1}}{X_{2} \omega \kappa_{1}}
$$

This condition may be interpreted by such way: the steady-state is stable, if:

- The diffusion of an alkali is less quick than the monomer diffusion (high values of $\kappa_{1}$ comparing to $\kappa_{2}$ (so, during the electropolymerization in organic solvents, like acetonitrile, the steady-state will be more stable, than in water, which may be confirmed by experimental data expressed in revision article ${ }^{6}$.

The electropolymerization rate is higher than the adsorption rate $\left(X_{2}>X_{1}\right)$, but with the great number of active adsorption sights (high value of $\xi$ );

The electropolymerization process does not affect the DEL or if this effect isn't too big $\left(\mathrm{X}_{2}>0\right)$. 
The last stable steady-state requirement is common for all the cases of electropolymerization ${ }^{25-34}$. The high value of $\mathrm{pH}$ of the pre-surface layer, at the same time, favors the steady-state stability (high value of $\mathrm{X}_{2}$ ).

As anodic electropolymerization is exotermic process, the diminishing of the temperature favors it, so the stability of steady-states will be controlled mostly by diffusion, which has to be rapid enough to maintain the steady-state. At higher temperature, the electropolymerization, adsorption and desorption are slower and the stability of steady-state is controlled mostly by them. It goes in accord to the data, described, for example, in ${ }^{6,9}$ and references therein.

If the diffusion of an alkali is more quick, than the formation of the conducting polymer, as an outlet for hydroxyl-ions (as dopants), the steady-state won't be stable. If the impacts of the mentioned factors are equal, the monotonic instability is to be realized. Its condition may be represented as:

$$
\kappa_{2}=\frac{X_{2} \kappa_{1}+X_{2} \xi-X_{1} \kappa_{1}}{X_{2} \omega \kappa_{1}}
$$

For the oscillatory behavior to be realized, it's ought (but isn't enough) for the main Jacobian diagonal to have positive elements. In the absence of autocatalytic hydroxyl formation (and it is the case), the only elements, capable to be positive are:

$X_{1}$ in the case of the attracting interaction of adsorbed molecules, supported by spin-spin and coulombic interaction. This cause is common for the systems with electropolymerization ${ }^{24-35}$ and confirms the presence of the surface instability, observed experimentally in ${ }^{10-18}$.

Cathodicelectrodeposition of polypyrrole has been recently realized for the first time ${ }^{35}$, by in situ generation of promoting $\mathrm{NO}^{+}$particles. The structure of the obtained polymer was slightly different, comparing to the obtained anodically from the same solution and the process wasn't accompanied by instabilities, which also confirms the relation "surface processes - electrochemical instabilities".

$-X_{2}$ in the case of the modification of the DEL by electropolymerization process. This influence is different in different solvent and in different electrolytes, so it explains the difference of the oscillation amplitude in different media, observed in ${ }^{10}$. This cause for the oscillatory $^{25-34}$ is also common for all the system with electropolymerization of heterocyclic compounds.

This model and its statements are valid for the case of furan, pyrrole and thiophene derivatives, whose polymerization potential is lower than the potential of hydroxyl oxidation, over the metals, inert in alkaline media. The changes made in the model for other cases are listed below:

\section{The model modification for other systems}

- Electropolymerization over activated alkali-soluble metals, forming hydroxyl complexes. As the alkali-soluble metals are of amphoteric nature (Al, Be, Ga, Zn etc.), their hydroxides are non-soluble in water, and the hydroxyl, formed by water reduction, is forming a film, that is then dissolved by solution hydroxyl to form a complex, for example:

$$
\mathrm{Zn}+2 \mathrm{NaOH}+2 \mathrm{H}_{2} \mathrm{O} \rightarrow \mathrm{Na}_{2}\left[\mathrm{Zn}(\mathrm{OH})_{4}\right]+\mathrm{H}_{2}
$$

Or

$$
2 \mathrm{Al}+6 \mathrm{NaOH}+6 \mathrm{H}_{2} \mathrm{O} \rightarrow 2 \mathrm{Na}_{3}\left[\mathrm{Al}(\mathrm{OH})_{6}\right]+3 \mathrm{H}_{2}
$$


As we suppose that the alkali concentration is enough to promote rapid film dissolution, we do not take in account its formation and in this case, the equation (4) is changed by

$$
\frac{d a}{d t}=\frac{2}{\delta}\left(\frac{D}{\delta}\left(a_{0}-a\right)-r_{2}-r_{3}\right)
$$

In which $r_{3}$ is the metal dissolution rate, that may be calculated as:

$$
r_{3}=k_{3} a^{\sigma}(1-\theta)
$$

in which $\sigma$ is alkali reaction order.

The analysis of the equation set $\left(2,34^{\prime}\right)$ may prove that in the absence of the passivation film, the possible oscillatory behavior is caused only by the factors of the electropolymerization (surface factor of the adsorbed monomer and the electrochemical factor of the electropolymerization in DEL). For the steady-state stability the metal dissolution is stabilizing factor, as it favors the diminishing of hydroxyl concentration.

Electropolymerizationof thiophene derivatives over palladium. As thiophene has higher potential that the potential of the oxidation of hydroxide, and taking in account that palladium doesn't react with oxygen forming, the equation is modified by nearly the same way

$$
\begin{gathered}
\frac{d a}{d t}=\frac{2}{\delta}\left(\frac{D}{\delta}\left(a_{0}-a\right)-r_{2}-r_{3}\right) \\
r_{3}=k_{3} a(1-\theta) \exp \left(\frac{4 F \varphi_{0}}{R T}\right)
\end{gathered}
$$

The analysis of the equation set $\left(2,34^{\prime \prime}\right)$ may prove that in the absence of the passivation film, the possible oscillatory behavior is caused only by the factors of the electropolymerization (surface factor of the adsorbed monomer and the electrochemical factor of the electropolymerization in DEL). For the steady-state stability the hydroxide oxidation is stabilizing factor, as it favors the diminishing of hydroxyl concentration (if it doesn't affects the DEL).

Electropolymerization of acid derivatives of heterocyclic compounds. As the monomer in this case is the furan, pyrrole and thiophene molecule, substituted by the group - $\mathrm{COOH}$ or $-\mathrm{SO}_{3} \mathrm{H}$, it is modified and the electropolymerization is realized by another compound, the salt of the mentioned acid. The equation set in this case becomes more complicated.

\section{Conclusions}

1. The presence of time dissipative structures, whose existence is maintained by the monomer and alkali diffusion and polymer formation.

2. The increasing of mobility of hydroxide ions, comparing to the monomer molecule, drives the steady-state off the stability region or approximates it to the stability margin. The high value of $\mathrm{pH}$, at the same time, favors the steady-state stability.

3. The absence of the influences of electrochemical reaction(s) on DEL favors the steadystate stability

4. The equality of impacts of alkali diffusion and its participation in electropolymerization leads to the monotonic instability.

5. The oscillatory instability, if any, may be caused by the same factors, as in other causes of electropolymerization. Possible metal dissolution and hydroxide oxidation don't cause it. 


\section{References}

1- T. Yamamoto, Asia Materials2, 2010, 54.

2- J. Mostany, B.R Scharifker. Electrochim. Acta, 1997, 42, 291.

3- J. Mostany, B.R Scharifker. Synth.Met., 1997, 87, 179.

4- $\quad$ S. Kumar.: E-J. Chem, 2010, 8, , 846.

5- $\quad$ Sharma L K, Kumar S, Singh S and Singh R K P, Russ.J.Electrochem, 2010, 46, , 37.

6- $\quad$ Sadki S. Scottland Ph., Brodie N., Saboraud G, Chem. Soc. Rev, 2000, 29, 283.

7- N. Bodnaryuk-Lupshak, O.I. Aksimentyeva, Vopr. Khim.Khim.Techn., 1998, 50, 1, published in Ukrainian

8- V.M. Nalwa. Handbook of Advanced Electronic and Photonic Materials and Devices, V8, Conducting polymers, Wiley \&Sons Ltd.- 1997.

9- J. Roncali.: Chem. Rev, 1992, 92, 711.

10- I.Das, N.R.Agrawal, S.A.Ansari, S.K.Gupta,Ind. J. Chem, 2008, 47º 1798.

11- S.U. Rahman, M.S. Ba-Shammakh, Synth. Met., 2004, 140, 207.

12- $\quad$ A.S.Liu, M.A.S. Oliveira. J. Braz. Chem Soc., 2007, 18, 143.

13- Sazou D., Synth. Met., 2002, 130, 45.

14- I.Das, N. Goel, N.R. Agrawal, S. K. Gupta, , J. Phys. Chem, 2010, 114, 12888.

15- M. Bazzaoui, E.A. Bazzaoui, L. Martins, J.I. Martins, Synth. Met., 2002, 130, 73.

16- I.Das, N. Goel, S. K. Gupta, N.R. Agrawal, J. Electroanal. Chem,2012. 670.

17- M. Iseki, Y. Sugiyama, US Patent US5608358 A, 1997, Sanyo Electric Co., Ltd. http://www.google.com/patents/US5608358.

18- Das, N.R. Agrawal, S.K.Gupta, R.P.Rastogi, J. Phys. Chem, 2009, 113, 5296.

19- T. Tatsuma, T. Ogawa, R. Sato, N. Oyama, J. Electroanal. Chem, 2001, 501, 180

20- $\quad$ K.Aoki, I. Mukoyama, J.Chen, Russ.J. Electrochem, 2004, 40, 319.

21- L. Hudson, M.R. Bassett, Rev. Chem. Eng, 1991, 7, 108.

22- $\quad$ M. Pagitsas, S. Dimitra, Electrochimica Acta, 1991, 36, 1301.

23- A.J. Pearlstein, J.A. Johnson, J. Electrochem. Soc, 1989, 136, 1290.

24- V.Tkach, V. Nechyporuk, P. Yagodynets, Ciên. Tecn. Mat, 2012, 24, 54.

25- $\quad$ V.Tkach, V. Nechyporuk, P. Yagodynets, Afinidad, 2013, 70, 73.

26- V.Tkach, V. Nechyporuk, P. Yagodynets, Avances en Química, 2013, 8, 9.

27- V. Tkach, V. Nechyporuk, P. Yagodynets', Tecn. Met. Mat. Min, 2013, 10, 249.

28- V. V. Tkach, V. V. Nechyporuk, P. I. Yagodynets', Geor. Chem. J., 2011, 11, 387.

29- V.V. Tkach, V.V. Nechyporuk, P.I. Yagodynets', S.C. de Oliveira, Al. M. Da Rocha, J. Sib. Fed. Univ. Chemistry., 2014, 7, 403, published in Russian.

30- V. V.Tkach, V. V.Nechyporuk, P. I. Yagodynets', Proceeding of the $3^{\text {rd }}$ Portuguese

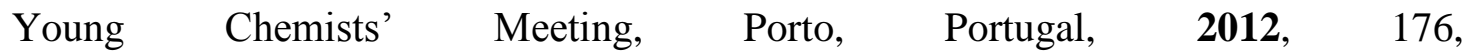
http://gqi.spq.pt/3pychem/images/Book_Abstracts_3PYCheM.pdf.

31- V. V. Tkach, V. V. Nechyporuk, P. I. Yagodynets', XXVI Congreso Peruano de Química Mariano Eduardo de Rivero y Ustáriz, Arequipa, Perú, 2012. 137.

32- V.V Tkach, V.V Nechyporuk., 4thInternational Symposium on Flexible Organic Electronics, Thessaloniki, 10thof July -14th of July, 2011, 148 .

33- V.V. Tkach, V.V. Nechyporuk, O.T. Slipenyuk, Ecl. Quím., 2012, $37,72$.

34- V.V. Tkach, V.V. Nechyporuk, Ecl. Quím., 2012, 37, 68.

35- Y.Jung, N. Singh, K.-Sh. Choi, Angew. Chem. Int. Ed., 2009, 48, 8331. 